\title{
Global Dynamics of a Virus Dynamical Model with Cell-to-Cell Transmission and Cure Rate
}

\author{
Tongqian Zhang, ${ }^{1,2}$ Xinzhu Meng, ${ }^{1,2}$ and Tonghua Zhang ${ }^{3}$ \\ ${ }^{1}$ College of Mathematics and Systems Science, Shandong University of Science and Technology, Qingdao 266590, China \\ ${ }^{2}$ State Key Laboratory of Mining Disaster Prevention and Control Co-Founded by Shandong Province and \\ the Ministry of Science and Technology, Shandong University of Science and Technology, Qingdao 266590, China \\ ${ }^{3}$ Department of Mathematics, Swinburne University of Technology, P.O. Box 218, Hawthorn, VIC 3122, Australia
}

Correspondence should be addressed to Xinzhu Meng; mxz721106@sdust.edu.cn

Received 16 May 2015; Revised 30 June 2015; Accepted 7 July 2015

Academic Editor: Konstantin Blyuss

Copyright (c) 2015 Tongqian Zhang et al. This is an open access article distributed under the Creative Commons Attribution License, which permits unrestricted use, distribution, and reproduction in any medium, provided the original work is properly cited.

\begin{abstract}
The cure effect of a virus model with both cell-to-cell transmission and cell-to-virus transmission is studied. By the method of next generation matrix, the basic reproduction number is obtained. The locally asymptotic stability of the virus-free equilibrium and the endemic equilibrium is considered by investigating the characteristic equation of the model. The globally asymptotic stability of the virus-free equilibrium is proved by constructing suitable Lyapunov function, and the sufficient condition for the globally asymptotic stability of the endemic equilibrium is obtained by constructing suitable Lyapunov function and using LaSalle invariance principal.
\end{abstract}

\section{Introduction and Model Formulation}

Since tobacco mosaic virus, the first virus of the world discovered by Beijerinck in 1898 [1], more and more viruses have been discovered by biologists, biomedical scientists, and medical scientists and more than 5,000 viruses have been recorded in detail [2]. However, according to a recent study, there are at least 32,0000 viruses waiting to be discovered in the spread between mammalian species. Identifying diseases caused by these viruses, especially those that can infect people, perhaps can help us to prevent epidemic disease [3]. At the early stage of the study, it is generally accepted that because of the specificity of viruses, virus can only infect certain plant or animal species; however, more and more cases associated with emerging zoonoses have appeared, and with a deeper understanding of the virus, we found that most viruses can infect humans, such as Human Immunodeficiency Virus (HIV), Prions, Influenza Virus, Rabies Virus, Ebola Virus, and Middle East Respiratory Syndrome Coronavirus (MERSV) [3-6].

Generally, the basic process of viral infection and virus replication occurs in six main steps: attachment, penetration, uncoating, replication, assembly, and release [7]. After the whole replicative cycle, free viruses begin to diffuse and infect new host cell. Therefore, investigating the processes of viral growth and destruction of host cells so as to gain the insights into the evolutionary processes of virus and cell in body is very important. To this end, mathematical models and analysis are powerful tools.

Since mathematical models and method of mathematical analysis were used to study the dynamics of the virus, lots of models have been established to explain the evolution of the uninfected target cells, infected cells, and the free virus. In these models, early works belonged to Nowak et al. [8], Nowak and May [9], Perelson and Nelson [10], and Perelson et al. [11]. The general class of models that have been studied [8-12] have a form similar to

$$
\begin{aligned}
& \frac{d x}{d t}=\Lambda-d x-\alpha v x, \\
& \frac{d y}{d t}=\alpha v x-a y, \\
& \frac{d v}{d t}=k y-u v,
\end{aligned}
$$


where $x, y$, and $v$ represent the concentrations of uninfected target cells, infected cells, and virus, respectively. For explanations of other parameters we refer to literature [12]. This model describes the processes of virus invading the target cells and the release of the virus due to the infected cell apoptosis. In the model, the authors use $\alpha v x$ to represent the interaction between uninfected target $x$ and virus $v$, which obey the principle of mass action. Based on model (1), more authors used nonlinear functions to describe the rate constant characterizing infection of cells, for example, $\beta x v /(x+y)$ in [13], $\beta x v /(1+b v)$ in $[14,15], \beta x v /(1+a x+b v)$ in [16], and $\beta x v /(1+a x+b v+a b x v)$ in [17], and for details of more general nonlinear incidence rate functions please see [18-20]. Notice that there exists a potentially possible cure rate of the infected cells to the susceptible host cells in the infection process of some virus, such as Hepatitis B Virus (HBV) [21-25] and HIV [26-32]; recently, Hattaf et al. [19] adopted a general nonlinear incidence rate function with the form $f(x, y, v) v$ and introduced cure rate (denoted by $\rho$ ) into the following model:

$$
\begin{aligned}
& \frac{d x}{d t}=\Lambda-d x-f(x, y, v) v+\rho y, \\
& \frac{d y}{d t}=f(x, y, v) v-(a+\rho) y, \\
& \frac{d v}{d t}=k y-u v .
\end{aligned}
$$

In model (2), $f(x, y, v)$ satisfies the following hypotheses:

$\left(H_{1}^{\prime}\right) f(0, y, v)=0$, for all $y \geq 0$.

$\left(H_{2}^{\prime}\right) \partial f(x, y, v) / \partial x>0$, for all $x>0, y \geq 0$, and $v \geq 0$.

$\left(H_{3}^{\prime}\right) \partial f(x, y, v) / \partial y \leq 0$ and $\partial f(x, y, v) / \partial v \geq 0$, for all $x \geq$ $0, y \geq 0$, and $v \geq 0$.

Recently, Tian and Liu [20] improved model (2) by proposing a more general nonlinear incidence rate function with the form $f(x, y, v)$ and investigated the following model:

$$
\begin{aligned}
& \frac{d x}{d t}=\Lambda-d x-f(x, y, v)+\rho y, \\
& \frac{d y}{d t}=f(x, y, v)-(a+\rho) y, \\
& \frac{d v}{d t}=k y-u v .
\end{aligned}
$$

In model (3), $f(x, y, v)$ satisfies the following hypotheses:

$\left(H_{1}\right) f(0, y, v)=0$, for all $y \geq 0$ and $v \geq 0$, and $f(x, y, 0)=$ 0 , for all $x \geq 0$ and $y \geq 0$.

$\left(H_{2}\right) \partial f(x, y, v) / \partial x>0$, for all $x \geq 0, y \geq 0$, and $v>0$.

$\left(H_{3}\right) \partial f(x, y, v) / \partial y \leq 0$, for all $x \geq 0, y \geq 0$, and $v \geq 0$.

$\left(H_{4}\right) \partial f(x, y, v) / \partial v \geq 0$ and $v(\partial f(x, y, v) / \partial v)-f(x, y, v) \leq$ 0 , for all $x \geq 0, y \geq 0$, and $v \geq 0$.

However, many researches show that direct cell-to-cell spread can happen in some enveloped viruses (e.g., Human
Immunodeficiency Virus type-1 (HIV-1) [33-37], Human T-Lymphotropic Virus Type-1 (HTLV-1) [38-41], Herpes Simplex Virus (HSV) [42], and Measles [43-45]). Cell-to-cell spread not only facilitates rapid viral dissemination, but may also promote immune evasion and influence disease [46]. Moreover, a recent study has shown that cell-to-cell spread of $\mathrm{HIV}-1$ can reduce the sensitivity to the antiretroviral drugs by multiple infections of target cells and, as a result, the efficacy of antiretroviral therapy is reduced [47].

Motivated by the works [18-20, 48], we propose a virus dynamical model with both cell-to-virus infection and cellto-cell transmission and cure rate as follows:

$$
\begin{aligned}
& \frac{d x}{d t}=\Lambda-d x-f(y, v) x+\rho y, \\
& \frac{d y}{d t}=f(y, v) x-(a+\rho) y, \\
& \frac{d v}{d t}=k y-u v,
\end{aligned}
$$

where $x, y$, and $v$ denote the number of host cells, infected cells, and free virus, respectively. And $d, a$, and $u$ are the death rates of them, respectively. Free virus is produced by infected cells at a rate $k y . \Lambda$ represents the regeneration rate of host cells. $\rho$ is the cure rate. $f(y, v) x=(\beta y+\alpha v) x$ represents the total infection rate of host cells, which is divided into two parts $\beta y x$ and $\alpha v x$. The former represents the part where infected cells infect host cells by direct contact, and the latter means that host cells are infected by the free virus. For more detail, please see [48]. In the present model, we can see $f(y, 0) x=\beta x y \not \equiv 0$, for all $x \geq 0$ and $y \geq 0$, and $\partial f(y, v) x / \partial y=\beta x \geq 0$ for all $x \geq 0, y \geq 0$, and $v \geq 0$, which do not satisfy conditions $\left(H_{3}^{\prime}\right)$ in model (2) and conditions $\left(H_{1}\right)$ and $\left(H_{3}\right)$ in model (3). For biological considerations, we will study system (4) in the closed set $A=\left\{(x, y, v) \in R_{+}^{3} \mid\right.$ $x+y \leq \Lambda / d, v \geq 0\}$.

The main goal of the present paper is to investigate the globally asymptotic stability of the equilibria of (4). This work is structured as follows. In Section 1, we give the motivation and study the background of the model. In Section 2, the existence of virus-free equilibrium and the endemic equilibrium is shown based on the basic reproduction number. And the local stability of the two equilibria is discussed in Section 3. We focus on the globally asymptotic stability of the two equilibria in Section 4. Finally, a brief conclusion and discussion are given in Section 5.

\section{Basic Reproduction Number and Equilibria}

The basic reproduction number $[49,50]$ of model $(4)$ is given as

$$
\mathscr{R}=\frac{\Lambda(\alpha k+\beta u)}{d u(a+\rho)} .
$$

Based on the basic reproduction number $\mathscr{R}$, we have Theorem 1. 
Theorem 1. Model (4) always has a virus-free equilibrium $E_{0}=\left(x_{0}, 0,0\right)$, where $x_{0}=\Lambda / d$. If $\mathscr{R}>1$, model (4) has a unique endemic equilibrium $E_{1}\left(x^{*}, y^{*}, v^{*}\right)$, where

$$
\begin{aligned}
x^{*} & =\frac{\Lambda}{d \mathscr{R}}, \\
y^{*} & =\frac{\Lambda}{a}\left(1-\frac{1}{\mathscr{R}}\right), \\
v^{*} & =\frac{k}{u} y^{*} .
\end{aligned}
$$

\section{Local Stability of the Two Equilibria}

In this section, we shall show the local stability of equilibria $E_{0}$ and $E_{1}$.

Theorem 2. For model (4), we have the following conclusion:

(i) $E_{0}$ is locally stable if $\mathscr{R}<1$ and unstable if $\mathscr{R}>1$.

(ii) $E_{1}$ is locally stable if $\mathscr{R}>1$.

Proof. We firstly prove (i). Notice the Jacobian of model (4) evaluated $E_{0}$ is given by

$$
J\left(E_{0}\right)=\left(\begin{array}{ccc}
-d & \rho-\beta x_{0} & -\alpha x_{0} \\
0 & \beta x_{0}-(a+\rho) & \alpha x_{0} \\
0 & k & -u
\end{array}\right)
$$

Obviously, $J\left(E_{0}\right)$ has an eigenvalue $\lambda=-\mu<0$, and the other two eigenvalues $\lambda_{2}$ and $\lambda_{3}$ satisfy

$$
\begin{aligned}
\lambda_{2}+\lambda_{3} & =-\left(a+\rho-\beta x_{0}+u\right) \\
& =-\left(\frac{\alpha k}{u \mathscr{R}}+\beta x_{0}\left(\frac{1}{\mathscr{R}}-1\right)+u\right), \\
\lambda_{2} \lambda_{3} & =\left(a+\rho-\beta x_{0}\right) u-\alpha k x_{0} \\
& =(a+\rho) u(1-\mathscr{R}) .
\end{aligned}
$$

Then, when $\mathscr{R}<1, \lambda_{2}+\lambda_{3}<0$, and $\lambda_{2} \lambda_{3}>0$, all the eigenvalues of $J\left(E_{0}\right)$ have negative real parts and $E_{0}$ is locally asymptotically stable. And when $\mathscr{R}>1$ and $\lambda_{2} \lambda_{3}<0, J\left(E_{0}\right)$ has a positive eigenvalue and $E_{0}$ is unstable.

Next, we prove (ii). The Jacobian of model (4) evaluated $E_{1}$ is

$$
J\left(E_{1}\right)=\left(\begin{array}{ccc}
-d-\alpha v^{*}-\beta y^{*} & \rho-\beta x^{*} & -\alpha x^{*} \\
\alpha v^{*}+\beta y^{*} & \beta x^{*}-(a+\rho) & \alpha x^{*} \\
0 & k & -u
\end{array}\right) \text {, }
$$

from which we have the characteristic equation

$$
A \lambda^{3}+B \lambda^{2}+C \lambda+D=0
$$

where

$$
\begin{aligned}
A= & u x^{*}, \\
B= & \rho y^{*} u+\Lambda u+x^{*} u^{2}+x^{* 2} \alpha k \\
C= & \Lambda \alpha x^{*} k+\Lambda u^{2}+\rho y^{*} \alpha x^{*} k+\rho y^{*} u^{2}-y^{*} u a \rho \\
& -y^{*} u \rho^{2}+y^{*} u a \beta x^{*}+y^{*} u \rho \beta x^{*}, \\
D= & y^{*} u a \alpha x^{*} k+y^{*} u^{2} a \beta x^{*}+y^{*} u^{2} \rho \beta x^{*}-y^{*} u^{2} a \rho \\
& -y^{*} u^{2} \rho^{2}+y^{*} u \rho \alpha x^{*} k .
\end{aligned}
$$

Obviously, $A, B>0$. And noticing that $x^{*}(u \beta+\alpha k)=u(a+\rho)$, we have

$$
\begin{aligned}
& C=\Lambda \alpha x^{*} k+\Lambda u^{2}+\rho y^{*} \alpha x^{*} k+\rho y^{*} u^{2}-y^{*} u a \rho \\
& -y^{*} u \rho^{2}+y^{*} u a \beta x^{*}+y^{*} u \rho \beta x^{*}=\Lambda \alpha x^{*} k+\Lambda u^{2} \\
& +\rho y^{*} u^{2}+y^{*} u a \beta x^{*}+\rho y^{*} x^{*}(\alpha k+u \beta) \\
& -\rho y^{*} u(a+\rho)=\Lambda \alpha x^{*} k+\Lambda u^{2}+\rho y^{*} u^{2} \\
& +y^{*} u a \beta x^{*}>0 \\
& D=y^{*} u a \alpha x^{*} k+y^{*} u^{2} a \beta x^{*}+y^{*} u^{2} \rho \beta x^{*}-y^{*} u^{2} a \rho \\
& -y^{*} u^{2} \rho^{2}+y^{*} u \rho \alpha x^{*} k=y^{*} u a \alpha x^{*} k+y^{*} u^{2} a \beta x^{*} \\
& +\rho u y^{*} x^{*}(u \beta+\alpha k)-\rho y^{*} u^{2}(a+\rho) \\
& =y^{*} u a \alpha x^{*} k+y^{*} u^{2} a \beta x^{*}>0, \\
& B C-A D=\left(\rho y^{*} u+\Lambda u+x^{*} u^{2}+x^{* 2} \alpha k\right) \\
& \cdot\left(\Lambda \alpha x^{*} k+\Lambda u^{2}+\rho y^{*} u^{2}+y^{*} u a \beta x^{*}\right) \\
& -u x^{*}\left(y^{*} u a \alpha x^{*} k+y^{*} u^{2} a \beta x^{*}\right) \\
& =\left(\rho y^{*} u+\Lambda u+x^{* 2} \alpha k+x^{*} u^{2}\right) \\
& \cdot\left(\Lambda \alpha x^{*} k+y^{*} u a \beta x^{*}+\Lambda u^{2}+\rho y^{*} u^{2}\right) \\
& -u x^{*}\left(y^{*} u a \alpha x^{*} k+y^{*} u^{2} a \beta x^{*}\right) \\
& =\left(\rho y^{*} u+\Lambda u+x^{* 2} \alpha k\right) \\
& \cdot\left(\Lambda \alpha x^{*} k+y^{*} u a \beta x^{*}+\Lambda u^{2}+\rho y^{*} u^{2}\right) \\
& +x^{*} u^{2}\left(\Lambda u^{2}+\rho y^{*} u^{2}\right) \\
& +x^{*} u^{2}\left(\Lambda \alpha x^{*} k+y^{*} u a \beta x^{*}\right) \\
& -u x^{*}\left(y^{*} u a \alpha x^{*} k+y^{*} u^{2} a \beta x^{*}\right) \\
& =\left(\rho y^{*} u+\Lambda u+x^{* 2} \alpha k\right) \\
& \cdot\left(\Lambda \alpha x^{*} k+y^{*} u a \beta x^{*}+\Lambda u^{2}+\rho y^{*} u^{2}\right)
\end{aligned}
$$




$$
\begin{aligned}
& +x^{*} u^{2}\left(\Lambda u^{2}+\rho y^{*} u^{2}\right)+x^{*} u^{2} \Lambda \alpha x^{*} k \\
& -u x^{*} y^{*} u a \alpha x^{*} k=\left(\rho y^{*} u+\Lambda u+x^{* 2} \alpha k\right) \\
& \cdot\left(\Lambda \alpha x^{*} k+y^{*} u a \beta x^{*}+\Lambda u^{2}+\rho y^{*} u^{2}\right) \\
& +x^{*} u^{2}\left(\Lambda u^{2}+\rho y^{*} u^{2}\right)+x^{* 2} k u^{2} \alpha\left(\Lambda-y^{*} a\right)>0,
\end{aligned}
$$

where $\mathscr{R}>1$ and $y^{*}=\Lambda / a(1-1 / \mathscr{R})<\Lambda / a$ are used. Then, by the Routh-Hurwitz Criterion [51], we know that all the roots of (10) always have negative real parts. Thus, the epidemic equilibrium $E_{1}$ is locally asymptotically stable for $\mathscr{R}>1$.

\section{Global Stability of the Two Equilibria}

In this section, we study the global behaviors of model (4) by constructing Lyapunov functions. Firstly, we show the global stability of $E_{0}$.

Theorem 3. If $\mathscr{R}<1$, the virus-free equilibrium $E_{0}$ is globally asymptotically stable.

Proof. Define a Lyapunov function $L_{1}(t)$ on $A$ as follows:

$$
\begin{aligned}
L_{1}= & x-x_{0}-x_{0} \ln \frac{x}{x_{0}}+\frac{\rho}{2(d+a) x_{0}}\left(x-x_{0}+y\right)^{2} \\
& +y+p v
\end{aligned}
$$

here, $p>0$ is a constant to be determined. It follows from (4) and (13) that

$$
\begin{aligned}
& \frac{d L_{1}}{d t}=\Lambda-d x-(\beta y+\alpha v) x+\rho y-\frac{x_{0}}{x}(\Lambda-d x \\
& -(\beta y+\alpha v) x+\rho y)+\frac{\rho}{2(d+a) x_{0}} 2\left(x-x_{0}+y\right) \\
& \cdot(\Lambda \\
& -d x-(\beta y+\alpha v) x+\rho y+(\beta y+\alpha v) x \\
& -(a+\rho) y)+(\beta y+\alpha v) x-(a+\rho) y+p(k y \\
& -u v)=d\left(x_{0}-x\right)-\frac{d x_{0}}{x}\left(x_{0}-x\right)+\rho y+(\beta y \\
& +\alpha v) x_{0}-\frac{x_{0}}{x} \rho y+\frac{\rho}{(d+a) x_{0}}\left(x-x_{0}+y\right) \\
& \cdot\left(d\left(x_{0}-x\right)-a y\right)-(a+\rho) y+p(k y \\
& -u v)=-\frac{d}{x}\left(x_{0}-x\right)^{2}+(\beta y+\alpha v) x_{0}+\rho y-\frac{x_{0}}{x} \\
& \cdot \rho y+\frac{\rho}{(d+a) x_{0}}\left(-d\left(x-x_{0}\right)^{2}\right.
\end{aligned}
$$

$$
\begin{aligned}
& \left.+(a+d) y\left(x_{0}-x\right)-a y^{2}\right)-(a+\rho) y+p(k y \\
& -u v)=-\frac{d}{x}\left(x_{0}-x\right)^{2}+(\beta y+\alpha v) x_{0}-\frac{\rho y}{x x_{0}}\left(x_{0}\right. \\
& -x)^{2}-\frac{d \rho}{(d+a) x_{0}}\left(x-x_{0}\right)^{2}-\frac{a \rho}{(d+a) x_{0}} y^{2}-(a \\
& +\rho) y+p(k y-u v)=-\frac{d}{x}\left(x_{0}-x\right)^{2}-\frac{\rho y}{x x_{0}}\left(x_{0}\right. \\
& -x)^{2}-\frac{d \rho}{(d+a) x_{0}}\left(x-x_{0}\right)^{2}-\frac{a \rho}{(d+a) x_{0}} y^{2} \\
& +k\left(p-\frac{a+\rho-\beta x_{0}}{k}\right) y+u\left(\frac{\alpha x_{0}}{u}-p\right) v .
\end{aligned}
$$

Since $\mathscr{R}<1$, we have $(\beta k+\alpha u) x_{0}<u(a+\rho)$; then, we can choose $p>0$ such that $\beta x_{0} / u<p<\left(a+\rho-\alpha x_{0}\right) / k$. Hence, we have that $d L_{1}(t) / d t<0$. Then, $E_{0}$ is globally asymptotically stable.

Next, we study the global stability of the endemic equilib$\operatorname{rium} E_{1}$.

Theorem 4. If $1<\mathscr{R} \leq 1+\delta$, the epidemic equilibrium $E_{1}$ is globally asymptotically stable, where $\delta=(\beta \Lambda+(a-\rho) d+$ $\sqrt{\left.(\beta \Lambda+(a-\rho) d)^{2}+4 a \rho d^{2}\right)} / 2 \rho d$.

Proof. If $\mathscr{R}>1$, we define a Lyapunov function $L_{2}(t)$ as follows:

$$
\begin{aligned}
L_{2}(t)= & x-x^{*}-x^{*} \ln \frac{x}{x^{*}}+\left(y-y^{*}-y^{*} \ln \frac{y}{y^{*}}\right) \\
& +\frac{\alpha x^{*} v^{*}}{k y^{*}}\left(v-v^{*}-v^{*} \ln \frac{v}{v^{*}}\right) \\
& +\frac{\rho}{2(d+a)}\left(x-x^{*}+y-y^{*}\right)^{2} .
\end{aligned}
$$

It follows from (4) and (15) that

$$
\begin{aligned}
& \frac{d L_{2}(t)}{d t}=\Lambda-d x-(\beta y+\alpha v) x+\rho y \\
& -\frac{x^{*}}{x}(\Lambda-d x-(\beta y+\alpha v) x+\rho y)+(\beta y+\alpha v) x \\
& -(a+\rho) y-\frac{y^{*}}{y}((\beta y+\alpha v) x-(a+\rho) y) \\
& +\frac{\alpha x^{*} v^{*}}{k y^{*}}\left(k y-u v-\frac{v^{*}}{v}(k y-u v)\right) \\
& +\frac{\rho}{2(d+a) x^{*}} 2\left(x-x^{*}+y-y^{*}\right) \\
& \cdot(\Lambda-d x+\rho y-(a+\rho) y)=-d \frac{\left(x-x^{*}\right)^{2}}{x}
\end{aligned}
$$




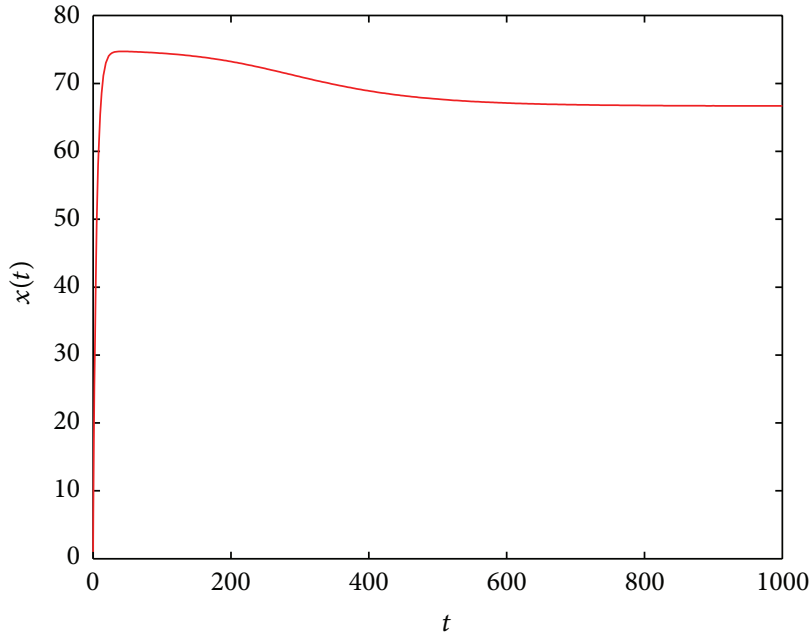

(a) Time series of $x(t)$

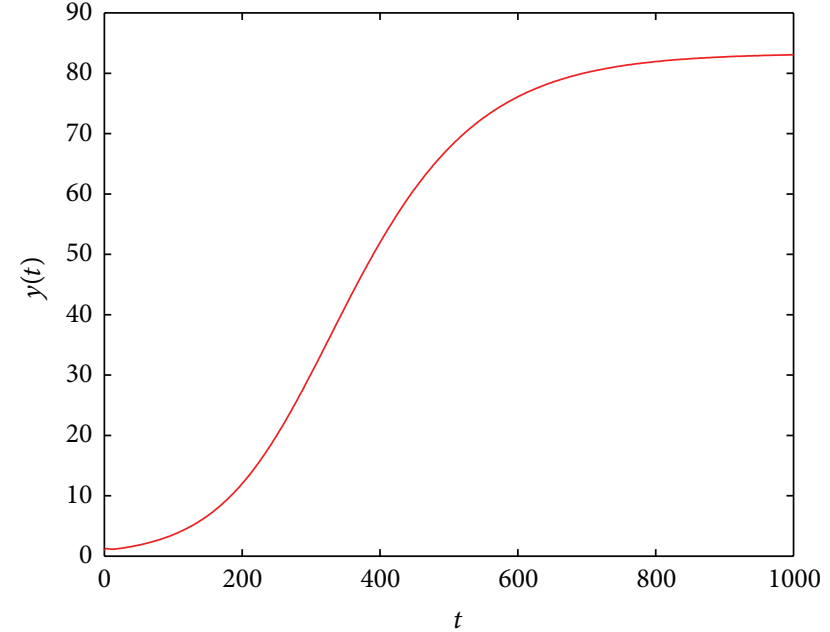

(b) Time series of $y(t)$

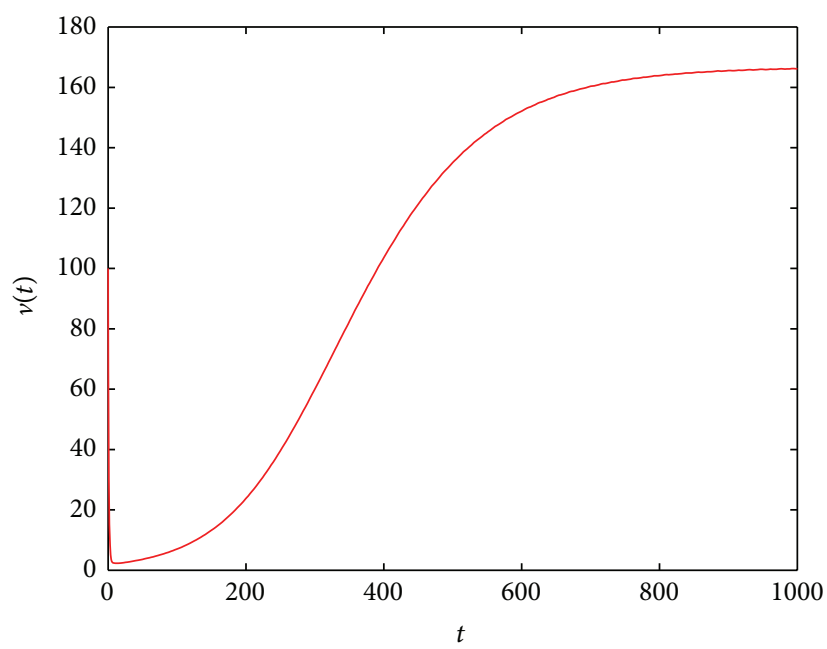

(c) Time series of $v(t)$

Figure 1: Illustration of numerical solution of system (4) with $\lambda=15, d=0.2, \beta=0.0008, \alpha=0.0005, \rho=0.1, a=0.02, k=2$, and $u=1$, and $x(0)=1, y(0)=1$, and $v(0)=100$. By calculation, one gets that $\mathscr{R}=1.125$ and $\delta=0.3583$; it is easy to verify $1<\mathscr{R}=1.125<1+\delta=1.3583$; then, the equilibrium $E_{1}$ is globally asymptotically stable.

$$
\begin{array}{ll}
+\beta x^{*} y^{*}+\alpha x^{*} v^{*}-\rho y^{*}+\rho y-\frac{x^{*}}{x} \beta x^{*} y^{*}-\frac{x^{*}}{x} & +\alpha x^{*} v^{*}\left(2-\frac{x^{*}}{x}+\frac{v}{v^{*}}-\frac{y^{*} v x}{x^{*} v^{*} y}-\frac{y}{y^{*}}\right) \\
\cdot \alpha x^{*} v^{*}+\frac{x^{*}}{x} \rho y^{*}+x^{*} \beta y+x^{*} \alpha v-\frac{x^{*}}{x} \rho y & +\alpha x^{*} v^{*}\left(\frac{y}{y^{*}}-\frac{v}{v^{*}}-\frac{v^{*} y}{v y^{*}}+1\right)+\frac{\rho}{x}\left(x-x^{*}\right) \\
-(a+\rho) y-y^{*} \beta x-\frac{y^{*}}{y} \alpha v x+\beta x^{*} y^{*}+\alpha x^{*} v^{*} & \cdot\left(y-y^{*}\right)-\frac{d \rho}{(d+a) x^{*}}\left(x-x^{*}\right)^{2} \\
+\frac{\alpha x^{*} v^{*}}{k y^{*}}\left(k y-u v-\frac{v^{*}}{v}(k y-u v)\right) & -\frac{a \rho}{(d+a) x^{*}}\left(y-y^{*}\right)^{2}+\frac{\rho}{x^{*}}\left(x-x^{*}\right)\left(y^{*}-y\right) \\
-\frac{d \rho}{(d+a) x^{*}}\left(x-x^{*}\right)^{2}-\frac{a \rho}{(d+a) x^{*}}\left(y-y^{*}\right)^{2} & =-d \frac{\left(x-x^{*}\right)^{2}}{x}-\beta y^{*} \frac{\left(x-x^{*}\right)^{2}}{x} \\
+\frac{\rho}{x^{*}}\left(\left(x-x^{*}\right)\left(y^{*}-y\right)\right)=-d \frac{\left(x-x^{*}\right)^{2}}{x}-\beta y^{*} & +\alpha x^{*} v^{*}\left(3-\frac{x^{*}}{x}-\frac{y^{*} v x}{x^{*} v^{*} y}-\frac{v^{*} y}{v y^{*}}\right) \\
. \frac{\left(x-x^{*}\right)^{2}}{x} & -\frac{d \rho}{(d+a) x^{*}\left(x-x^{*}\right)^{2}-\frac{a \rho}{(d+a) x^{*}}\left(y-y^{*}\right)^{2}}
\end{array}
$$




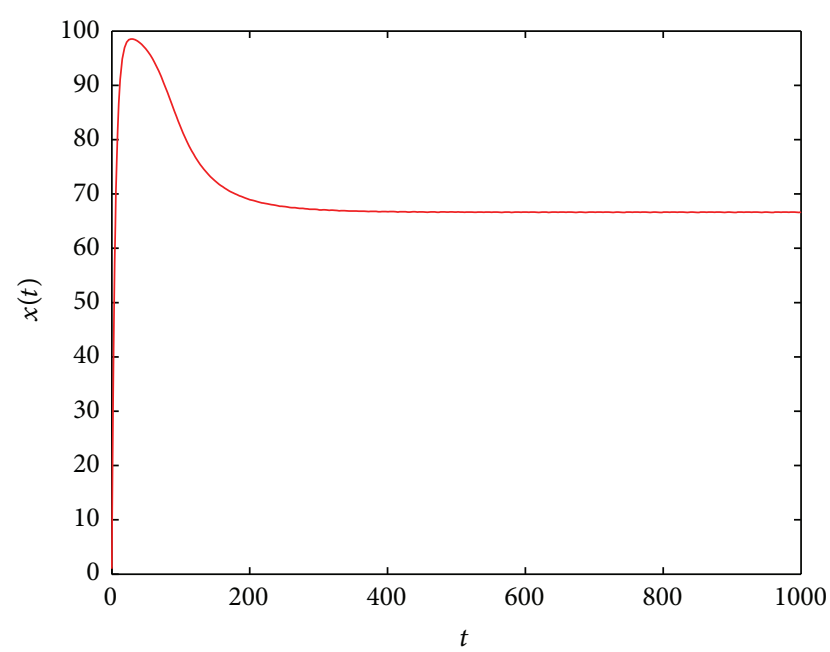

(a) Time series of $x(t)$

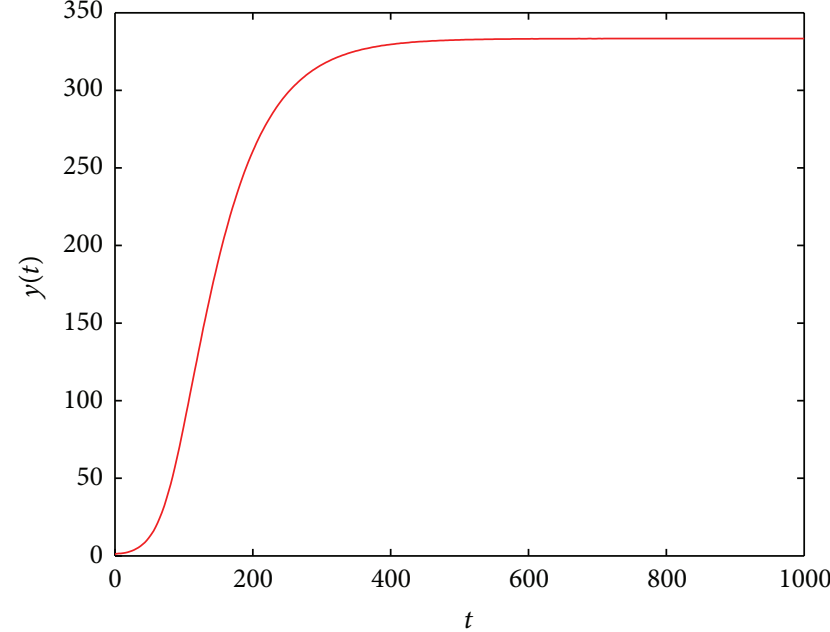

(b) Time series of $y(t)$

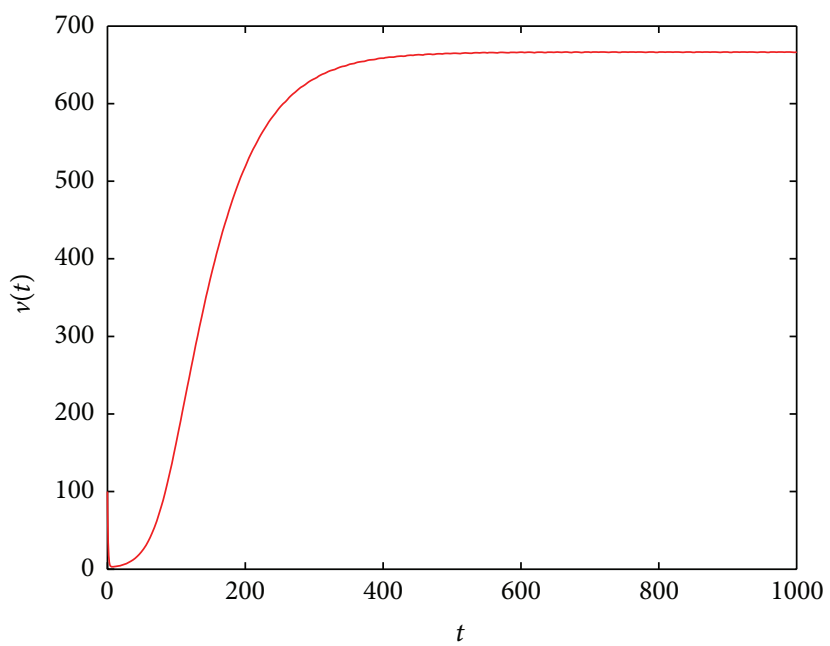

(c) Time series of $v(t)$

Figure 2: Illustration of numerical solution of system (4) with $\lambda=20, d=0.2, \beta=0.0008, \alpha=0.0005, \rho=0.1, a=0.02, k=2$, and $u=1$, and $x(0)=1, y(0)=1$, and $v(0)=100$. By calculation, one gets that $\mathscr{R}=1.5$ and $\delta=0.4472$; it is easy to verify $1<\mathscr{R}=1.5>1+\delta=1.4472$, while the equilibrium $E_{1}$ is also globally asymptotically stable.

$$
\begin{aligned}
& -\frac{\rho}{x x^{*}}\left(x-x^{*}\right)^{2}\left(y-y^{*}\right) \\
& =-\left(d x^{*}+\frac{d \rho x}{d+a}+\rho\left(y-y^{*}\right)+\beta x^{*} y^{*}\right) \\
& \cdot \frac{\left(x-x^{*}\right)^{2}}{x x^{*}}+\alpha x^{*} v^{*}\left(3-\frac{x^{*}}{x}-\frac{y^{*} v x}{x^{*} v^{*} y}-\frac{v^{*} y}{v y^{*}}\right) \\
& -\frac{a \rho}{(d+a) x^{*}}\left(y-y^{*}\right)^{2} \\
& =-\left(d x^{*}+\beta x^{*} y^{*}-\rho y^{*}+\frac{d \rho x}{d+a}+\rho y\right) \\
& \cdot \frac{\left(x-x^{*}\right)^{2}}{x x^{*}}+\alpha x^{*} v^{*}\left(3-\frac{x^{*}}{x}-\frac{y^{*} v x}{x^{*} v^{*} y}-\frac{v^{*} y}{v y^{*}}\right) \\
& -\frac{a \rho}{(d+a) x^{*}}\left(y-y^{*}\right)^{2} .
\end{aligned}
$$

Since the arithmetic mean is greater than or equal to the geometric mean, it follows that

$$
3-\frac{x^{*}}{x}-\frac{y^{*} v x}{x^{*} v^{*} y}-\frac{v^{*} y}{v y^{*}} \leq 0 .
$$

The above equality holds only for $x=x^{*}, y=y^{*}$, and $v=v^{*}$. Clearly, if $\mathscr{R}>1$ and $d x^{*}-\beta x^{*} y^{*}-\rho y^{*}>0$, then $d L_{2}(t) / d t \leq 0$. Note that $d x^{*}-\beta x^{*} y^{*}-\rho y^{*} \geq 0$ can be formulated as

$$
\begin{aligned}
1< & \mathscr{R} \\
\leq & 1 \\
& \quad+\frac{\beta \Lambda+(a-\rho) d+\sqrt{(\beta \Lambda+(a-\rho) d)^{2}+4 a \rho d^{2}}}{2 \rho d} \\
& \quad 1+\delta .
\end{aligned}
$$


Since $d L_{2}(t) / d t=0$ if and only if $x=x^{*}, y=y^{*}$, and $v=$ $v^{*}$, by LaSalle invariance principle [52], the equilibrium $E_{1}$ is globally asymptotically stable.

\section{Conclusion and Discussion}

In this paper, we considered the cure effect of a virus model with both cell-to-cell transmission and cell-to-virus transmission. By the method of next generation matrix, the basic reproduction number $\mathscr{R}$ is obtained. Firstly the locally asymptotic stability of the virus-free equilibrium and the endemic equilibrium is considered. Then, the globally asymptotic stability of the virus-free equilibrium is proved by constructing suitable Lyapunov function, and the sufficient condition for the globally asymptotic stability of the endemic equilibrium is obtained by constructing suitable Lyapunov function and using LaSalle invariance principal. By analyzing the condition for the globally asymptotic stability of the endemic equilibrium, we have that if $\rho=0$, from Theorem 4 , the conditions $\mathscr{R}>1$ can ensure the global stability of the equilibrium $E_{1}$, While if $\rho>0$, by the numerical simulations (see Figures 1 and 2), we find that $\mathscr{R} \leq 1+\delta$ in Theorem 4 is not necessary and can be dropped.

\section{Conflict of Interests}

The authors declare that there is no conflict of interests regarding the publication of this paper.

\section{Acknowledgments}

Tongqian Zhang and Xinzhu Meng are supported by the National Natural Science Foundation of China (no. 11371230), Shandong Provincial Natural Science Foundation, China (no. ZR2012AM012, ZR2015AQ001), a Project for Higher Educational Science and Technology Program of Shandong Province of China (no. J13LI05), Joint Innovative Center for Safe and Effective Mining Technology and Equipment of Coal Resources, Shandong Province of China, and SDUST Research Fund (2014TDJH102).

\section{References}

[1] M. W. Beijerinck, "Über ein Contagium vivum fluidum als Ursache der Fleckenkrankheit der Tabaksblätter," in Verhandelingen der Koninklyke akademie van Wettenschappen te Amsterdam, vol. 65, pp. 1-22, 1898, (German), Translated into English: J. Johnson, Ed., Phytopathological Classics, no. 7, pp. 33-52, American Phytopathological Society, St. Paul, Minn, USA, 1942.

[2] M. Breitbart and F. Rohwer, "Here a virus, there a virus, everywhere the same virus?" Trends in Microbiology, vol. 13, no. 6, pp. 278-284, 2005.

[3] S. J. Anthony, J. H. Epstein, K. A. Murray et al., "A strategy to estimate unknown viral diversity in mammals," mBio, vol. 4, no. 5, Article ID e00598-13, 2013.

[4] World Health Organization, Zoonoses and the Human-AnimalEcosystems Interface, WHO, 2014.

[5] L. H. Taylor, S. M. Latham, and M. E. J. Woolhouse, "Risk factors for human disease emergence," Philosophical Transactions of the Royal Society B: Biological Sciences, vol. 356, no. 1411, pp. 983-989, 2001.

[6] P. A. Marx, C. Apetrei, and E. Drucker, "AIDS as a zoonosis? Confusion over the origin of the virus and the origin of the epidemics," Journal of Medical Primatology, vol. 33, no. 5-6, pp. 220-226, 2004.

[7] L. Collier, A. Balows, and M. Sussman, Topley and Wilson's Microbiology and Microbial Infections, vol. 1 of Virology, Arnold, 9th edition, 1998, edited by: B. Mahy, L. Collier.

[8] M. A. Nowak, S. Bonhoeffer, A. M. Hill, R. Boehme, H. C. Thomas, and H. Mcdade, "Viral dynamics in hepatitis B virus infection," Proceedings of the National Academy of Sciences of the United States of America, vol. 93, no. 9, pp. 4398-4402, 1996.

[9] M. A. Nowak and R. M. May, Viral Dynamics, Oxford University Press, Oxford, UK, 2000.

[10] A. S. Perelson and P. W. Nelson, "Mathematical analysis of HIV1 dynamics in vivo," SIAM Review, vol. 41, no. 1, pp. 3-44, 1999.

[11] A. S. Perelson, A. U. Neumann, M. Markowitz, J. M. Leonard, and D. D. Ho, "HIV-1 dynamics in vivo: virion clearance rate, infected cell life-span, and viral generation time," Science, vol. 271, no. 5255, pp. 1582-1586, 1996.

[12] P. W. Nelson and A. S. Perelson, "Mathematical analysis of delay differential equation models of HIV-1 infection," Mathematical Biosciences, vol. 179, no. 1, pp. 73-94, 2002.

[13] L. Min, Y. Su, and Y. Kuang, "Mathematical analysis of a basic virus infection model with application to HBV infection," Rocky Mountain Journal of Mathematics, vol. 38, no. 5, pp. 1573-1585, 2008.

[14] D. Li and W. Ma, "Asymptotic properties of a HIV-1 infection model with time delay," Journal of Mathematical Analysis and Applications, vol. 335, no. 1, pp. 683-691, 2007.

[15] X. Song and A. U. Neumann, "Global stability and periodic solution of the viral dynamics," Journal of Mathematical Analysis and Applications, vol. 329, no. 1, pp. 281-297, 2007.

[16] G. Huang, W. Ma, and Y. Takeuchi, "Global properties for virus dynamics model with Beddington-DeAngelis functional response," Applied Mathematics Letters, vol. 22, no. 11, pp. 16901693, 2009.

[17] X. Zhou and J. Cui, "Global stability of the viral dynamics with Crowley-Martin functional response," Bulletin of the Korean Mathematical Society, vol. 48, no. 3, pp. 555-574, 2011.

[18] G. Huang, Y. Takeuchi, and W. Ma, "Lyapunov functionals for delay differential equations model of viral infections," SIAM Journal on Applied Mathematics, vol. 70, no. 7, pp. 2693-2708, 2010.

[19] K. Hattaf, N. Yousfi, and A. Tridane, "Mathematical analysis of a virus dynamics model with general incidence rate and cure rate," Nonlinear Analysis: Real World Applications, vol. 13, no. 4, pp. 1866-1872, 2012.

[20] Y. Tian and X. Liu, "Global dynamics of a virus dynamical model with general incidence rate and cure rate," Nonlinear Analysis: Real World Applications, vol. 16, no. 1, pp. 17-26, 2014.

[21] L. G. Guidotti, R. Rochford, J. Chung, M. Shapiro, R. Purcell, and F. V. Chisari, "Viral clearance without destruction of infected cells during acute HBV infection," Science, vol. 284, no. 5415, pp. 825-829, 1999.

[22] K. Hattaf and N. Yousfi, "Hepatitis B virus infection model with logistic hepatocyte growth and cure rate," Applied Mathematical Sciences, vol. 5, no. 45-48, pp. 2327-2335, 2011.

[23] K. Wang, A. Fan, and A. Torres, "Global properties of an improved hepatitis B virus model," Nonlinear Analysis: Real World Applications, vol. 11, no. 4, pp. 3131-3138, 2010. 
[24] H. Dahari, E. Shudo, R. M. Ribeiro, and A. S. Perelson, "Modeling complex decay profiles of hepatitis B virus during antiviral therapy," Hepatology, vol. 49, no. 1, pp. 32-38, 2009.

[25] Q. Huan, P. Ning, and W. Ding, "Global stability for a dynamic model of hepatitis B with antivirus treatment," Journal of Applied Analysis and Computation, vol. 3, no. 1, pp. 37-50, 2013.

[26] X. Jiang, X. Zhou, X. Shi, and X. Song, "Analysis of stability and Hopf bifurcation for a delay-differential equation model of HIV infection of CD $4^{+}$T-cells," Chaos, Solitons and Fractals, vol. 38, no. 2, pp. 447-460, 2008.

[27] X. Zhou, X. Song, and X. Shi, "A differential equation model of HIV infection of $\mathrm{CD} 4^{+}$T-cells with cure rate," Journal of Mathematical Analysis and Applications, vol. 342, no. 2, pp. 1342-1355, 2008.

[28] P. K. Srivastava and P. Chandra, "Modeling the dynamics of HIV and $\mathrm{CD} 4^{+} \mathrm{T}$ cells during primary infection," Nonlinear Analysis: Real World Applications, vol. 11, no. 2, pp. 612-618, 2010.

[29] K. Hattaf and N. Yousf, "Dynamics of HIV infection model with therapy and cure rate," International Journal of Tomography \& Statistics, vol. 16, no. 11, pp. 74-80, 2011.

[30] X. Liu, H. Wang, Z. Hu, and W. Ma, "Global stability of an HIV pathogenesis model with cure rate," Nonlinear Analysis: Real World Applications, vol. 12, no. 6, pp. 2947-2961, 2011.

[31] Y. Xiao, H. Miao, S. Tang, and H. Wu, "Modeling antiretroviral drug responses for HIV-1 infected patients using differential equation models," Advanced Drug Delivery Reviews, vol. 65, no. 7, pp. 940-953, 2013.

[32] J. Li, X. Song, and F. Gao, "Global stability of a virus infection model with two delays and two types of target cells," Journal of Applied Analysis and Computation, vol. 2, no. 3, pp. 281-292, 2012.

[33] D. McDonald, L. Wu, S. M. Bohks, V. N. KewalRamani, D. Unutmaz, and T. J. Hope, "Recruitment of HIV and its receptors to dendritic cell-T cell junctions," Science, vol. 300, no. 5623, pp. 1295-1297, 2003.

[34] Q. Sattentau, "Avoiding the void: cell-to-cell spread of human viruses," Nature Reviews Microbiology, vol. 6, no. 11, pp. 815-826, 2008.

[35] B. S. Stein, S. D. Gowda, J. D. Lifson, R. C. Penhallow, K. G. Bensch, and E. G. Engleman, "pH-independent HIV entry into CD4-positive $\mathrm{T}$ cells via virus envelope fusion to the plasma membrane," Cell, vol. 49, no. 5, pp. 659-668, 1987.

[36] D. M. Phillips, "The role of cell-to-cell transmission in HIV infection,” AIDS, vol. 8, no. 6, pp. 719-731, 1994.

[37] H. Sato, J. Orensteint, D. Dimitrov, and M. Martin, “Cell-to-cell spread of HIV-1 occurs within minutes and may not involve the participation of virus particles," Virology, vol. 186, no. 2, pp. 712724, 1992.

[38] T. Igakura, J. C. Stinchcombe, P. K. C. Goon et al., "Spread of HTLV-I between lymphocytes by virus-induced polarization of the cytoskeleton," Science, vol. 299, no. 5613, pp. 1713-1716, 2003.

[39] A.-M. Pais-Correia, M. Sachse, S. Guadagnini et al., "Biofilmlike extracellular viral assemblies mediate HTLV-1 cell-to-cell transmission at virological synapses," Nature Medicine, vol. 16, no. 1, pp. 83-89, 2010.

[40] C. R. M. Bangham, "The immune control and cell-to-cell spread of human T-lymphotropic virus type 1," Journal of General Virology, vol. 84, no. 12, pp. 3177-3189, 2003.

[41] Y. Satou, J.-I. Yasunaga, M. Yoshida, and M. Matsuoka, "HTLV-I basic leucine zipper factor gene mRNA supports proliferation of adult T cell leukemia cells," Proceedings of the National Academy of Sciences of the United States of America, vol. 103, no. 3, pp. $720-725,2006$.

[42] K. S. Dingwell, C. R. Brunetti, R. L. Hendricks et al., "Herpes simplex virus glycoproteins $\mathrm{E}$ and I facilitate cell-to-cell spread in vivo and across junctions of cultured cells," Journal of Virology, vol. 68, no. 2, pp. 834-845, 1994.

[43] W. P. Duprex, S. McQuaid, L. Hangartner, M. A. Billeter, and B. K. Rima, "Observation of measles virus cell-to-cell spread in astrocytoma cells by using a green fluorescent proteinexpressing recombinant virus," Journal of Virology, vol. 73, no. 11, pp. 9568-9575, 1999.

[44] S. Krantic, C. Gimenez, and C. Rabourdin-Combe, "Cell-tocell contact via measles virus haemagglutinin-CD46 interaction triggers CD46 downregulation," Journal of General Virology, vol. 76, no. 11, pp. 2793-2800, 1995.

[45] T. F. Wild, E. Malvoisin, and R. Buckland, "Measles virus: both the haemagglutinin and fusion glycoproteins are required for fusion," Journal of General Virology, vol. 72, no. 2, pp. 439-442, 1991.

[46] C. Jolly, "Cell-to-cell transmission of retroviruses: innate immunity and interferon-induced restriction factors," Virology, vol. 411, no. 2, pp. 251-259, 2011.

[47] A. Sigal, J. T. Kim, A. B. Balazs et al., "Cell-to-cell spread of HIV permits ongoing replication despite antiretroviral therapy," Nature, vol. 477, no. 7362, pp. 95-99, 2011.

[48] X. Lai and X. Zou, "Modeling HIV-1 virus dynamics with both virus-to-cell infection and cell-to-cell transmission," SIAM Journal on Applied Mathematics, vol. 74, no. 3, pp. 898-917, 2014.

[49] O. Diekmann, J. A. P. Heesterbeek, and J. A. Metz, "On the definition and the computation of the basic reproduction ratio $R_{0}$ in models for infectious diseases in heterogeneous populations," Journal of Mathematical Biology, vol. 28, no. 4, pp. 365-382, 1990.

[50] P. Van den Driessche and J. Watmough, "Reproduction numbers and sub-threshold endemic equilibria for compartmental models of disease transmission," Mathematical Biosciences, vol. 180, no. 1, pp. 29-48, 2002.

[51] E. J. Routh, W. K. Clifford, C. Sturm, and M. Bocher, Stability of Motion, Taylor \& Francis, London, UK, 1975.

[52] J. P. La Salle, The Stability of Dynamica Systems, SIAM Publications, Philadelphia, Pa, USA, 1976. 


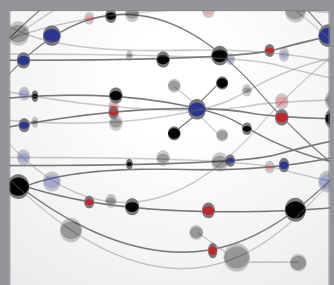

The Scientific World Journal
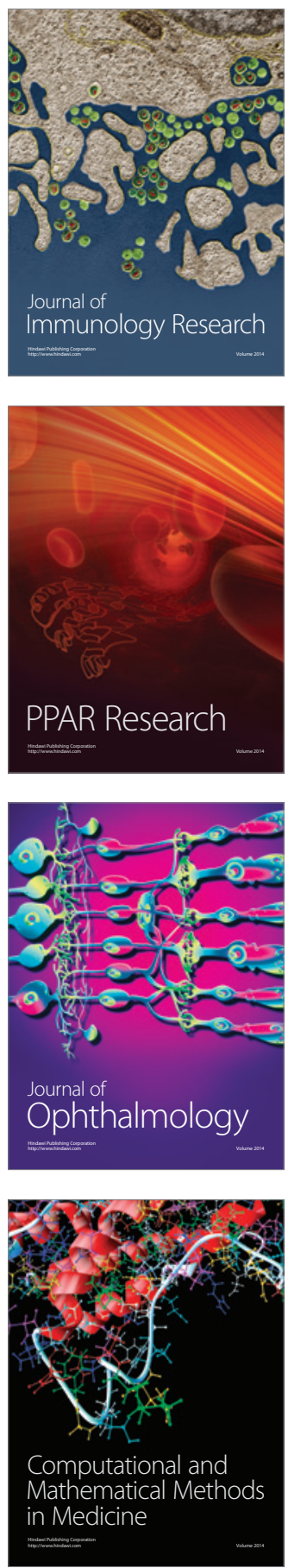

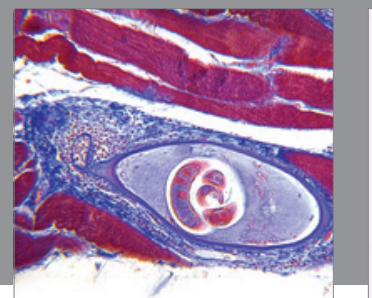

Gastroenterology

Research and Practice
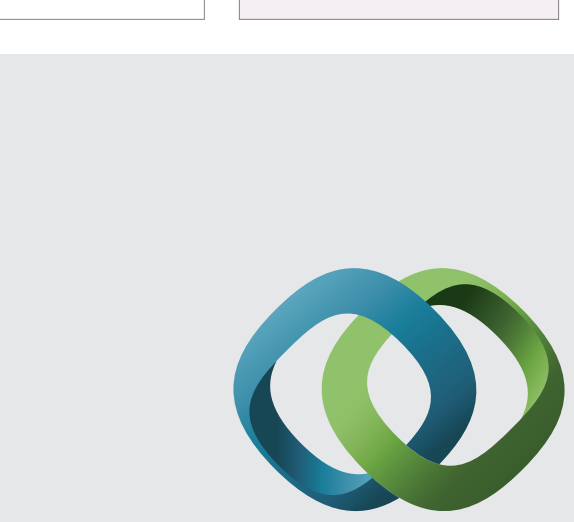

\section{Hindawi}

Submit your manuscripts at

http://www.hindawi.com
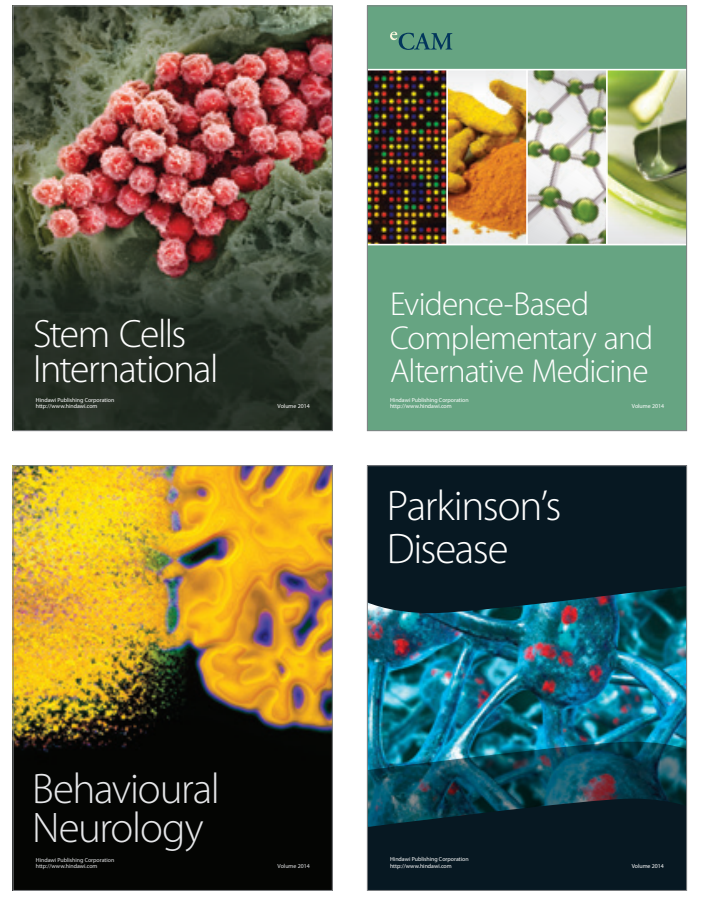
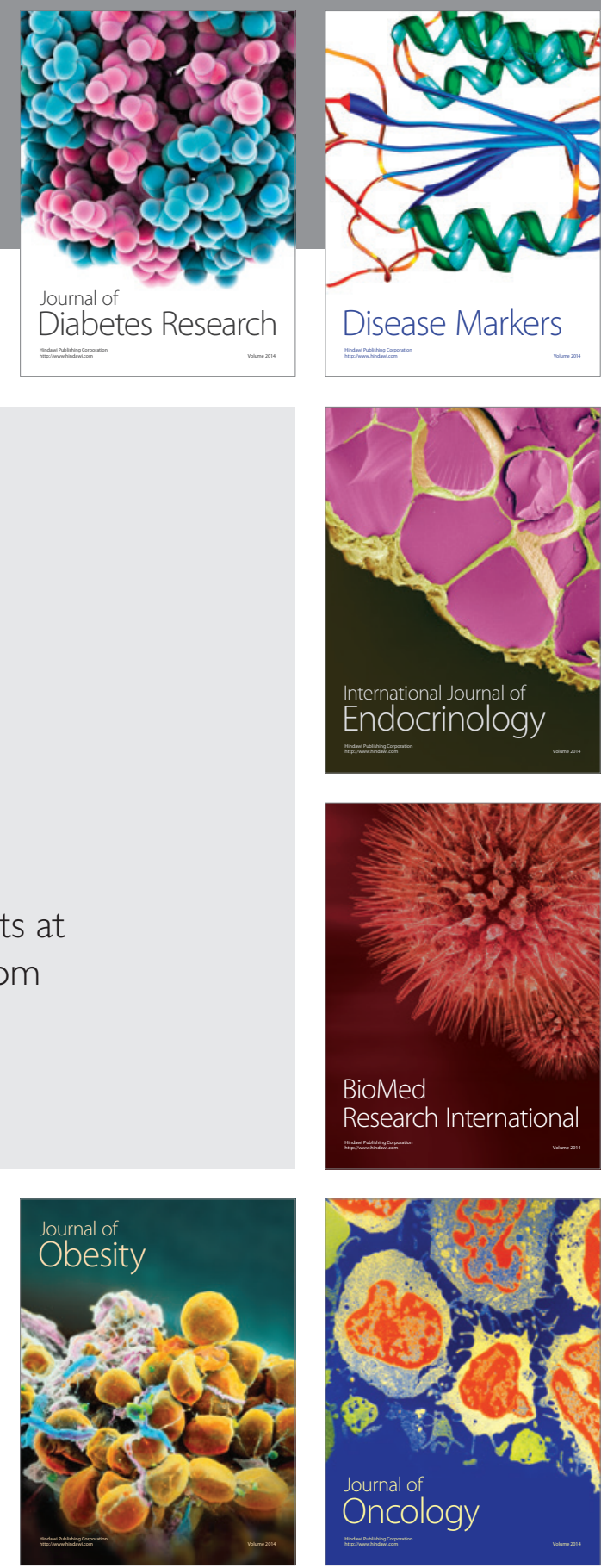

Disease Markers
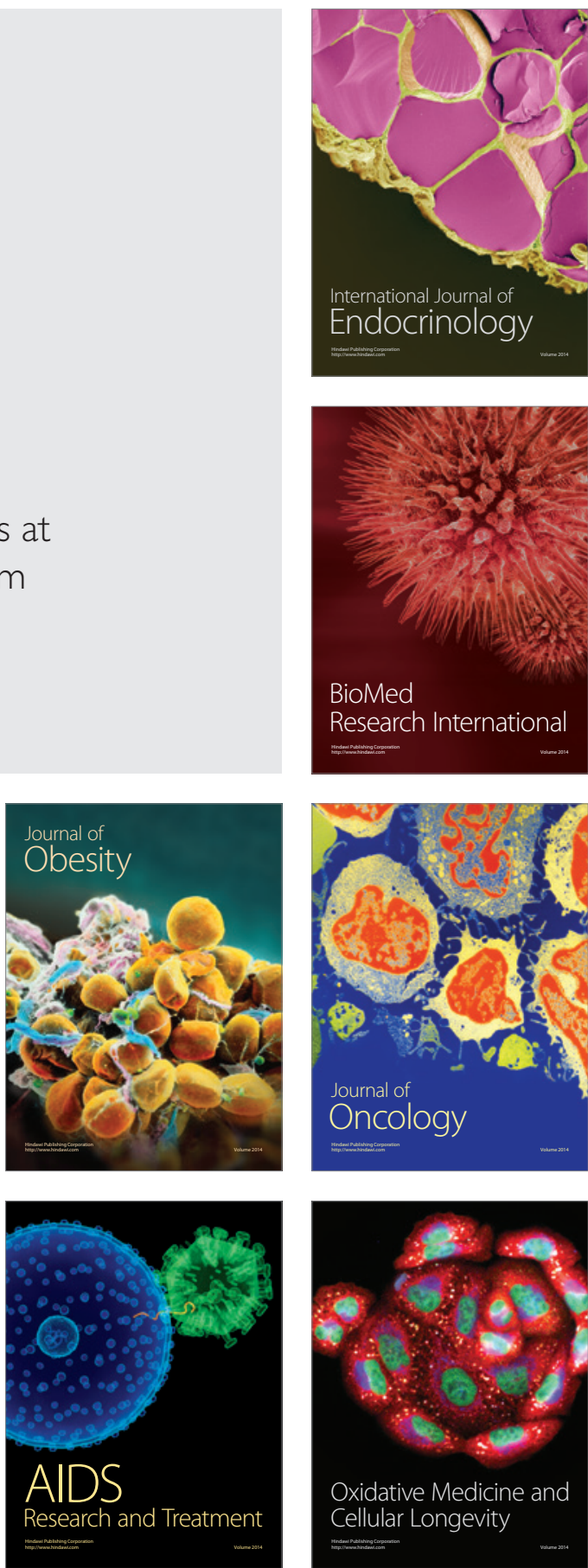